\title{
Diagnostik und Therapie der chronischen nicht-infektiösen Diarrhö
}

Korinna Ulbricht, Peter Layer, Viola Andresen

\begin{abstract}
Diarrhö gehört zu den 20 häufigsten Beratungsanlässen in der Hausarztpraxis [1]. Pro Jahr treten in Deutschland rund 65 Mio. Episoden einer akuten gastrointestinalen Erkrankung auf. Die Mehrzahl davon ist infektiöser Genese [2]. Davon abzugrenzen ist die chronische Diarrhö mit einer Dauer über 3 Wochen. Sie kann Symptom unterschiedlicher gastrointestinaler und systemischer Störungen sein [3].
\end{abstract}

Definition | Diarrhö bedeutet

- die Stuhlfrequenz nimmt auf mehr als dreimal täglich zu und / oder

- die Stuhlmenge beträgt mehr als $200 \mathrm{~g} / \mathrm{d}$ und

- die Konsistenz ist vermindert (Stuhlwassergehalt $>80 \%$ ).

Beeinflusst werden diese drei Parameter durch den Ballaststoffgehalt der Nahrung, Medikamente, Stress und Extremsport (z. B. Marathonlauf) $[3,4]$.

Zur Objektivierung anamnestischer Angaben eignen sich die Erstellung eines Stuhlprotokolls und die Erfassung der Stuhlkonsistenz z.B. mittels „Bristol-Stool-Form-Scale“ ( $\triangleright$ Abb. 1). Es konnte gezeigt werden, dass mehr Patienten mit organischen Erkrankungen einer Diagnostik zugeführt wurden, wenn die Kriterien von Typ 6 und Typ 7 der Bristol-Stool-Form-Scale zu den klassischen Definitionskriterien der Diarrhö hinzugezogen wurden [5].

Ursachen | Verschiedene Mechanismen, die häufig miteinander vergesellschaftet sind, führen zu einer (chronischen) Diarrhö:

- Die verminderte Resorption (Malabsorption) des Lumeninhaltes führt zu osmotischer Diarrhö, die vor allem postprandial auftritt.

- Um eine sekretorische Diarrhö handelt es sich, wenn eine aktive Flüssigkeitssekretion der Schleimhaut stattfindet, wie z.B. bei chologener Diarrhö, peptidproduzierenden Tumoren oder villösem Kolonadenom.

- Bei Erkrankungen, die mit intestinalen Motilitätsstörungen einhergehen, spricht man von motorischer Diarrhö.

- Im Rahmen von entzündlichen Schleimhautveränderungen mit Schädigung der Mukosa wie bei Morbus Crohn und Colitis ulcerosa können exsudative Diarrhöen auftreten.

Osmotische vs. sekretorische Diarrhö | Um zwischen osmotischer und sekretorischer Diarrhö zu unterscheiden, kann die anamnestische Frage nach Sistieren der Beschwerden unter Nahrungs- karenz weiterhelfen. Oft haben die Patienten bereits diesbezügliche Beobachtungen gemacht. Es besteht die Möglichkeit eines 24-Stunden-Hungerversuchs (keine Nahrungsaufnahme über $24 \mathrm{~h}$, Trinken von Wasser sowie kalorien- und koffeinfreien Getränken möglich). Sistieren hierunter die Beschwerden, liegt sehr wahrscheinlich eine osmotische Diarrhö vor. Eine sekretorische Diarrhö würde hingegen fortbestehen.

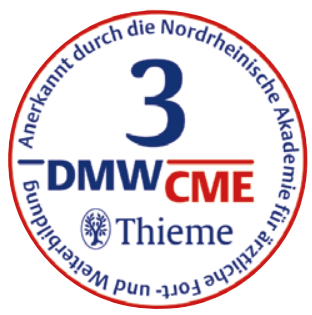

\section{Differenzialdiagnostik der chronischen nicht-infektiösen Diarrhö}

Anamnese I Neben einer allgemeinen internistischen Anamnese sollten folgende Faktoren gezielt erfragt werden:

- Beginn bzw. Auslöser der Symptomatik,

- tageszeitliches Auftreten,

- Fluktuation der Symptomatik,

- Zusammenhang mit bestimmten Nahrungsmitteln,

- Flush,

- Vormedikation,

- Alkohol- und Nikotinkonsum,

- abdominelle Voroperationen und

- Vor- bzw. Grunderkrankungen.

Abb. 1 Spezielle Stuhlanamnese: Die Bristol-Stool-FormScale.

\section{Typ Beschreibung \\ 1 Getrennte Klumpen wie Nüsse (schwierig zu entleeren) \\ 2 Wurstform, aber klumpig \\ 3 Wurstform, aber mit Rissen und Spalten auf der Oberfläche \\ 4 Wurst- oder Schlangeform, weich und glatt \\ 5 Weiche Häufchen mit klaren Rändern (leicht zu entleeren) \\ 6 Flockige Stücke mit zerrissenen Rändern, breiiger Stuhl \\ 7 Wässrig, keine festen Bestandteile, vollkommen flüssig}

Form

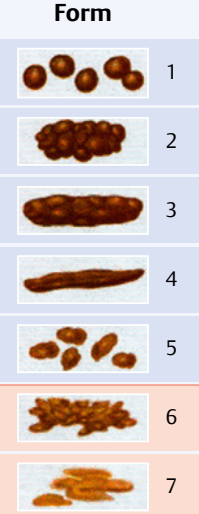

Typ $1+2=$ Obstipation, Typ 3-5=normaler Stuhl, Typ $6+7=$ Diarrhoe 


\begin{tabular}{|c|c|}
\hline Diarrhö-Form & Differenzialdiagnose \\
\hline Motorische Diarrhö & $\begin{array}{l}\text { - funktionelle Diarrhö } \\
\text { - Diarrhö-dominantes Reizdarmsyndrom } \\
\text { - Hyperthyreose } \\
\text { - diabetische Diarrhö } \\
\text { - Karzinoidsyndrom } \\
\text { - Post-Vagotomie-Syndrom } \\
\text { - Amyloidose }\end{array}$ \\
\hline Osmotische Diarrhö & $\begin{array}{l}\text { - Laxanzien (Laktulose, magnesiumhaltige Präparate) } \\
\text { - Malabsorptionssyndrome }\end{array}$ \\
\hline $\begin{array}{l}\text { Malabsorptive } \\
\text { Diarrhö }\end{array}$ & $\begin{array}{l}\text { - Enzymdefekte (z. B. Laktasemangel) } \\
\text { - Zöliakie } \\
\text { - Exokrine Pankreasinsuffizienz } \\
\text { - Kurzdarmsyndrom } \\
\text { - } \text { bakterielle Fehlbesiedlung } \\
\text { - } \\
\text { Atrophie oder Infiltration der Mukosa (Skleroder- } \\
\text { mie, Amyloidose, Lymphom) } \\
\text { Medikamente (Zytostatika, NSAR-Kolitis, antibioti- } \\
\text { kassoziierte/pseudomembranöse Kolitis), } \\
\text { chemische Noxen } \\
\text { - } \text { mikroskopische Kolitis (kollagene/lymphozytäre } \\
\text { - Morbus Whipple } \\
\text { - } \text { Darminfektion (Bakterien, Viren, Parasiten) } \\
\text { - HIV-assoziierte Enteropathie }\end{array}$ \\
\hline Sekretorische Diarrhö & $\begin{array}{l}\text { - peptidproduzierende Tumoren (VIPom, Karzinoid, } \\
\text { Gastrinom, C-Zell-Karzinom) } \\
\text { - villöses Kolonadenom } \\
\text { - chologene Diarrhö (Idiopathisches Gallensäuren- } \\
\text { verlust-Syndrom, Z. n. Cholezystektomie, Vagoto- } \\
\text { mie, lleozökalresektion) }\end{array}$ \\
\hline Exsudative Diarrhö & $\begin{array}{l}\text { Entzündungen ( M. Crohn, Colitis ulcerosa) } \\
\text { - Ischämische Kolitis } \\
\text { - M. Whipple } \\
\text { - mikrobielle Entzündungen }\end{array}$ \\
\hline
\end{tabular}

Tab. 1 Wichtige Differenzialdiagnosen der chronischen Diarrhö. Einzelne Erkrankungen können mehrere Mechanismen bedingen; die Tabelle erhebt keinen Anspruch auf Vollständigkeit.
Allgemeine Diagnostik | Die klinische Untersuchung umfasst:

- Ernährungszustand(BMI),

- Auffälligkeiten des Abdomen (Resistenzen, Druckschmerz, auffällige Auskultation),

- rektale Untersuchung (Inkontinenz bei vermindertem Sphinktertonus),

- Stuhlinspektion,

- Lymphknotenschwellungen und

- extraintestinale Symptome (Gelenke, Haut- und Schleimhautveränderungen, Sehstörungen) Laborchemisch sollten zunächst Differenzialblutbild, Elektrolyte, C-reaktives Protein, Blutsenkungsgeschwindigkeit, INR, Eisen, Ferritin, TSH, Transaminasen, $y$-GT, Alkalische Phosphatase, Bilirubin, Lipase, Cholinesterase, LDH, Nierenretentionsparameter und ggf. Blutzucker sowie $\mathrm{HbA}_{1 \mathrm{c}}$ bestimmt werden. Bei anamnestisch möglicher Zöliakie werden Gewebstransglutaminase-IgAAntikörper (tTG-IgA-Ak) oder die EndomysiumIgA-Antikörper (EmA-IgA-Ak) sowie das GesamtIgA im Serum untersucht [6]. Bei Zeichen einer Malassimilation sollten zusätzlich Albumin, Gesamteiweiß, Vitamin $B_{12}$ und Folsäure bestimmt werden.

Stuhluntersuchungen | Ein erhöhtes fäkales Calprotectin oder Lactoferrin können hinweisend auf eine entzündliche Darmerkrankung sein [7]. Die Calprotectin-Konzentration im Stuhl ist direkt proportional zur Menge der im Rahmen einer Entzündungsreaktion in die Darmwand eingewanderten neutrophilen Granulozyten. Beim Zerfall der Granulozyten wird Calprotectin freigesetzt und mit dem Stuhl ausgeschieden [7-9]. Im Stuhl ist es bis zu einer Woche bei Raumtemperatur stabil und mittels ELISA messbar, da es durch Bakterien nicht abgebaut werden kann [10]. Fäkales Calprotectin ist ein nützlicher Marker zur Unterscheidung zwischen (chronisch) entzündlichen Darmerkrankungen und funktionellen Darmbeschwerden [11].

Cave! Ein normaler Calprotectinspiegel schließt eine entzündliche Darmerkrankung nicht aus!

- Gewichtsverlust (ungewollt)

- Leistungsknick

- Blut-, Schleim- oder Eiterbeimengungen im Stuhl

- Fieber

- Anämie

- Exsikkose

- Malnutritionszeichen

- neu aufgetretenen abdominelle Schmerzen

- nächtliche Diarrhoe

- heller/entfärbter Stuhl

- positive Familienanamnese für chronisch entzündliche Darmerkrankungen und (gastrointestinale) Malignome

Die wichtigsten Differenzialdiagnosen der chronischen Diarrhö sind in $\triangleright$ Tab. 1 zusammengefasst.
Eine verminderte Konzentration der PankreasElastase $1 \mathrm{im}$ Stuhl, insbesondere $<100 \mu \mathrm{g} / \mathrm{g}$ Stuhl, liefert einen Hinweis auf eine exokrine Pankreasinsuffizienz. Sie kann aber bei einer Diarrhö falsch erniedrigt sein und gilt nicht als beweisend [12]. Die Sensitivität der fäkalen Pankreas-Elastase 1 liegt zwischen 54\% bei leichter exokriner Pankreasinsuffizienz und $95 \%$ bei schwerer exokriner Pankreasinsuffizienz [13]. Alternativ kann ein ${ }^{13} \mathrm{C}$ -Triglycerid-Atemtest Hinweise auf eine gestörte intestinale Lipolyse liefern (s. unten).

Urinuntersuchung | Die Absorptionskapazität des Dünndarms kann mittels D-Xylose-Test überprüft werden. Nach oraler Gabe von $25 \mathrm{~g}$ des Mo- 
nosaccharids sollte bei normaler Absorption über den Dünndarm und normaler Nierenfunktion

- mehr als $4 \mathrm{~g}$ über $5 \mathrm{~h}$ im Urin ausgeschieden werden oder

- in einer Blutprobe nach 60 Minuten ein Spiegel von $>25 \mathrm{mg} / \mathrm{dl}$ erreicht werden.

In $20-30 \%$ ist der Test falsch positiv oder falsch negativ. Einnahme von NSAR, eine eingeschränkte Nierenfunktion, Magenentleerungsstörungen, portale Hypertension und Aszites beeinträchtigen die Aussagefähigkeit des Tests [14].

Sonografie I Es sollte besonders auf Zeichen einer chronischen Pankreatitis (Pseudozysten, Verkalkungen), die Darmperistaltik, Darmwandverdickungen/-perfusion, Erweiterungen und / oder Stenosen des Darmlumens bzw. Flüssigkeitsfüllung, Raumforderungen in Projektion auf Dickund Dünndarmschlingen (Neoplasien) und vergrößerte Lymphknoten geachtet werden. Bei sonografischen Auffälligkeiten oder nicht sicher zu interpretierenden, möglicherweise pathologischen Befunden ist ein zweites bildgebendes Verfahren sinnvoll. Je nach Fragestellung sollte eine möglichst gezielte Untersuchung, z. B. eine orale Endosonografie und / oder Magnetresonanz-Cholangiopankreatikografie (MR-CP) bei Auffälligkeiten im pankreatikobiliären System erfolgen. Um nach möglichen Dünndarmpathologien zu fahnden, eignet sich ein MR-Sellink (mit oralem und intravenösem Kontrastmittel) und bei spezifischem Verdacht ggf. eine Videokapselendoskopie zur Beurteilung der Dünndarmschleimhaut. Bei Verdacht auf eine viszerale Durchblutungsstörung helfen die Duplex-Sonografie und CT- oder MR-Angiografie weiter. Je nach anamnestischen Verdachtsdiagnosen und klinischer Konstellation sollte über die Abfolge des weiteren diagnostischen Vorgehens entschieden werden ( Abb. 2).

Ileokoloskopie | Im Rahmen der endoskopischen Diagnostik (Ileokoloskopie) bei Diarrhö sind zusätzlich zu gezielten Biopsien bei Schleimhautveränderungen und Raumforderungen die Entnahme von Biopsien im terminalen Ileum und Kolonstufenbiopsien zu empfehlen [15]. Neben der histologischen Untersuchung kann bereits das Verteilungsmuster von Schleimhautveränderungen Hinweise auf die Genese liefern:

- Colitis ulcerosa mit kontinuierlichem Schleimhautbefall,

- Morbus Crohn häufig segmental,

- ischämische Kolitis eher linksseitiges, seltener rechtsseitiges Hemikolon.

Ösophagogastroduodenoskopie | Duodenalbiopsien im Rahmen einer Ösophagogastroduodenoskopie dienen vor allem der Diagnose einer Zöliakie [6]. Seltenere Krankheitsbilder mit ähnlicher Symptomatik, die sich mittels Duodenalbiopsien erfassen lassen, sind Morbus Whipple, Giardiasis, Autoimmunenteropathien, genuine intestinale Lymphangiektasie (Morbus Wald- mann), sekundäre Lymphangiektasieformen und Amyloidose.

Funktionsdiagnostik mittels $\mathbf{H}_{2}$-Atemtest I Das Untersuchungsprinzip des $\mathrm{H}_{2}$-Atemtest beruht darauf, dass bei der Verdauung von Kohlenhydraten durch Darmbakterien unter anderem Wasserstoff freigesetzt, von der Darmmukosa resorbiert, über das Blut zur Lunge transportiert und dort abgeatmet wird. Gelangen Laktose oder Fruktose bei Laktasemangel oder verzögerter Fruktoseresorption in das Kolon, kommt es zu einem Anstieg von $\mathrm{H}_{2}$ in der Ausatemluft.

Bakterielle Fehlbesiedlung des Dünndarms | Bei eher unspezifischen abdominellen Beschwerden sollte an eine bakterielle Fehlbesiedlung des Dünndarms gedacht und ein $\mathrm{H}_{2}$-Glukose-Atemtest durchgeführt werden. Glukose wird schnell im proximalen Dünndarm resorbiert, bei bakterieller Fehlbesiedlung allerdings vor der Resorption unter anderem zu Wasserstoff metabolisiert. Eine bakterielle Fehlbesiedlung des Dünndarms kann ein falsch-positives Ergebnis im $\mathrm{H}_{2}$-Laktose-, -Fruktose- oder -Sorbitol-Atemtest bedingen und sollte deshalb differenzialdiagnostisch ausgeschlossen werden.

Andererseits produziert bei ca. 30 bis $40 \%$ der Menschen die Darmflora wenig oder kein $\mathrm{H}_{2}$ (niedriger Anteil an Anaerobiern), sodass falsch-negative Ergebnissen in den $\mathrm{H}_{2}$ Atemtests auftreten können [16].

Überprüft werden kann dies anhand eines $\mathrm{H}_{2}$ Laktulose-Atemtests. Das Disaccharid Laktulose wird im Dünndarm weder gespalten noch resorbiert, sondern im Dickdarm bakteriell zu molekularem Wasserstoff $\left(\mathrm{H}_{2}\right)$ metabolisiert. Sind keine $\mathrm{H}_{2}$-produzierenden Bakterien vorhanden (kann z.B. auch bis zu 4 Wochen nach Antibiotikaeinnahme und prograder Darmspülung auftreten) spricht man von einem $\mathrm{H}_{2}$-Non-Producer-Status. Bei vorhandenen $\mathrm{H}_{2}$-produzierenden Bakterien kann mithilfe des $\mathrm{H}_{2}$-Laktulose-Atemtest die orozökale Transitzeit gemessen werden. Das Anlegen einer Kultur aus endoskopisch entnommenem Jejunumaspirat wird häufig als Goldstandard der Diagnose einer bakteriellen Fehlbesiedlung des Dünndarms angesehen (pathologische Keimzahl $>10^{4} / \mathrm{ml}$ ), ist jedoch aufwendig und daher wenig gebräuchlich $[17,18]$.

Exokrine Pankreasinsuffizienz | Zur indirekten Testung der Pankreasfunktion, insbesondere bei Verdacht auf eine exokrine Pankreasinsuffizienz und zur Therapiekontrolle unter laufender Pankreasenzymsubstitution, eignet sich $\mathrm{der}{ }^{13} \mathrm{C}$-Triglycerid-Atemtest mit markierten gemischten Triglyzeriden in Form einer Testmahlzeit. Nach Spaltung im Darmlumen werden die Monoglyzeride bzw. die ${ }^{13} \mathrm{C}$-markierten Fettsäuren resorbiert und zu 


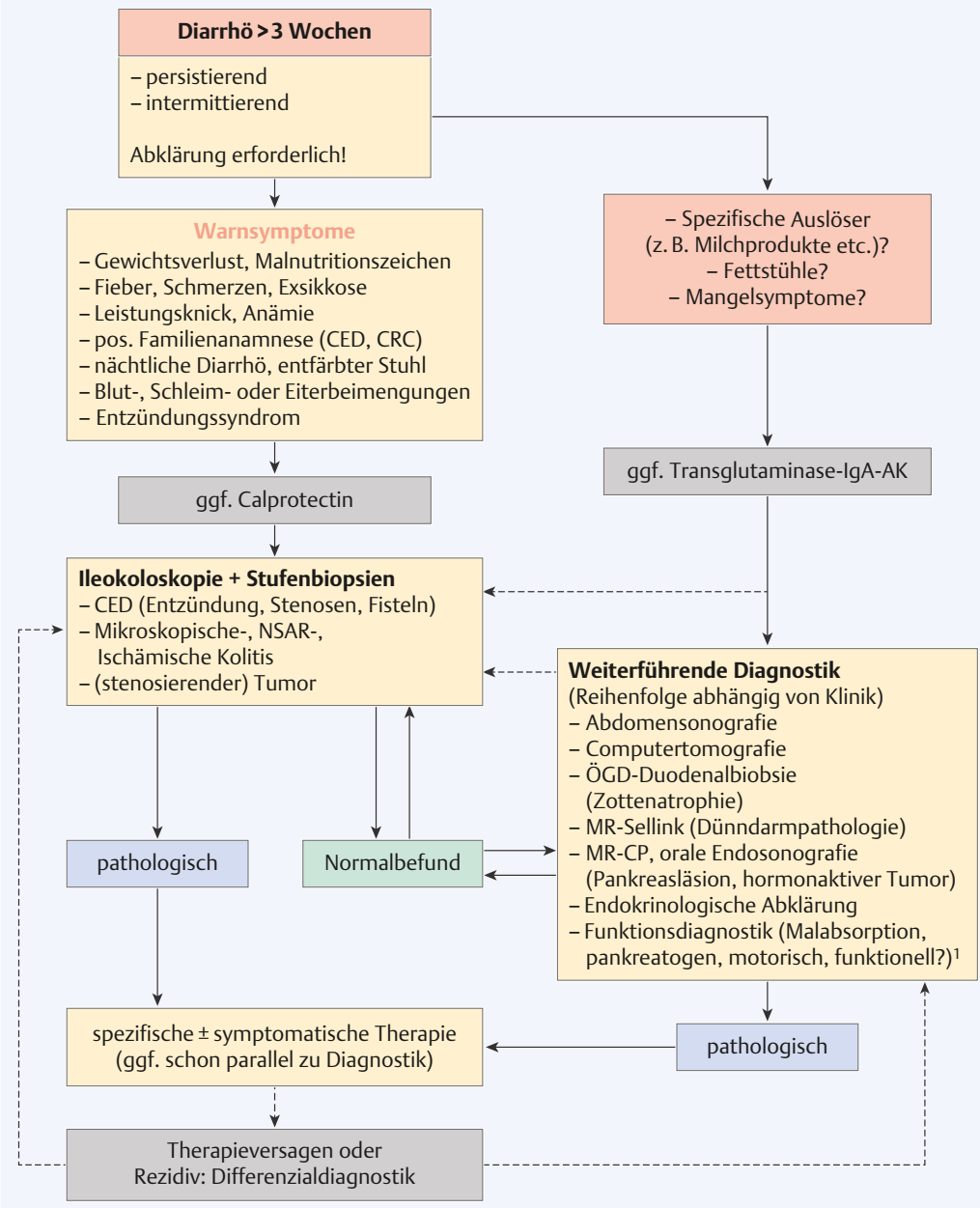

Abb. 2 Diagnostischer Algorithmus bei chronisch nicht-infektiöser Diarrhö.

${ }^{1}$ Funktionsdiagnostik: Testauswahl nach Symptomen $\left({ }^{13} \mathrm{C}\right.$-Triglyzerid-Atemtest, $\mathrm{H}_{2}$-Glukose-, -Laktulose-, -Laktose-, -Fruktose- und -Sorbitol-Atemtest sowie XyloseTest). und PYY, die die Pankreassekretion hemmen und den Appetit vermindern [20].

Gallensäureverlustsyndrom | Ein Gallensäureverlustsyndrom wird durch einen SeHCAT-Test (nuklearmedizinische Quantifizierung des Gallensäureverlustes) diagnostiziert. Bei sekundären Formen lässt sich die Diagnose bereits anamnestisch vermuten und wird in der Regel ohne diagnostische Testung durch eine Ex-juvantibus-Therapie mit Colestyramin gesichert [3].

Gynäkologische Abklärung | Frauen, die Symptome eines Reizdarms mit Diarrhö schildern, sollten gynäkologisch vorgestellt werden. Typische Symptome eines Reizdarms zählen auch zu den häufigen Erst- bzw. Frühsymptomen von Ovarialtumoren [15].

Spezialdiagnostik | Seltene Ursachen einer chronischen Diarrhö bedürfen einer Spezialdiagnostik, auf die hier nicht im Detail eingegangen werden kann. Dazu zählen

- Zollinger-Ellison-Syndrom (Gastrinspiegel, ggf. Sekretin-Provokationstest),

- neuroendokrine Tumoren (Chromogranin A),

- Karzinoide (5-Hydroxyindolessigsäure in 24hUrin),

- Kollagenosen (ANA, c- und p-ANCA),

- VIPome (VIPim Blut),

- Amyloidosen (Kongorot-Färbung der Biopsien),

- HIV-assoziierte Enteropathien,

- C-Zell-Karzinome,

- intesinale Lymphome,

- eosinophile Gastroenteritiden,

- systemische Sklerodermie,

- Vaskulitiden,

- systemische Mastozytose.

${ }^{13} \mathrm{CO}_{2}$ oxidiert. Der Anstieg von ${ }^{13} \mathrm{CO}_{2}$ in der Ausatemluft spiegelt somit indirekt die Lipaseaktivität im Darmlumen wider ( Abb. 3). Es wird hiermit allerdings der gesamte Fettverdauungsprozess abgebildet [19]. Die Sensitivität des ${ }^{13} \mathrm{C}$-TriglyceridAtemtest liegt bei $90-100 \%$ bei schwerer und bei $62-100 \%$ bei leichter exokriner Pankreasinsuffizienz, die Spezifität bei 80-90\% [13].

Asynchronie I Nach partieller Pankreatoduodenektomie kann es trotz ausreichender exokriner Parenchymmasse zu manifester Maldigestion kommen. Eine Ursache hierfür ist die postprandiale/ postcibale Asynchronie. Durch die Ableitung des Pankreassekrets in eine Jejunumschlinge, die von der Nahrung nicht passiert wird, folgt eine unzureichende Durchmischung von Speisebrei und Enzymen, sodass ein Teil der Nahrung unverdaut bleibt. Auch nach Gastrektomie kann es aufgrund einer beschleunigten Nahrungspassage zu einer postcibalen Asynchronie kommen [3]. Hinzu kommt die sogenannte „ileal break“, eine durch den raschen Durchtritt der Nahrung ins Ileum induzierte Freisetzung der Hormone GLP-1

\section{Allgemeine symptomatische Therapie}

Spezifische Therapie der zugrunde liegenden Erkrankungen | Auf die jeweilige spezifische Therapie der einzelnen Differenzialdiagnosen chronischer, nicht-infektiöser Diarrhö kann aufgrund des großen Umfangs im Folgenden nicht im Einzelnen eingegangen werden. Wir haben uns daher auf die symptomatische Therapie chronischer Diarrhö, Diarrhö bei Reizdarmsyndrom, funktioneller Diarrhö und der bakteriellen Fehlbesiedlung des Dünndarms konzentriert.

Ballaststoffe | Ballaststoffe, v.a. Gelbildner wie

- Plantago-afra/-ovata-Samenschalen,

- Hemicellulose und

- Pektine

können die Stuhlkonsistenz positiv beeinflussen. Die Gasbildung ist geringer als bei Faserstoffen (Weizenkleie, Leinsamen).

Loperamid I Der $\mu$-Opioid-Rezeptor-Agonist Loperamid (2 mg) sollte 45 Minuten vor den 

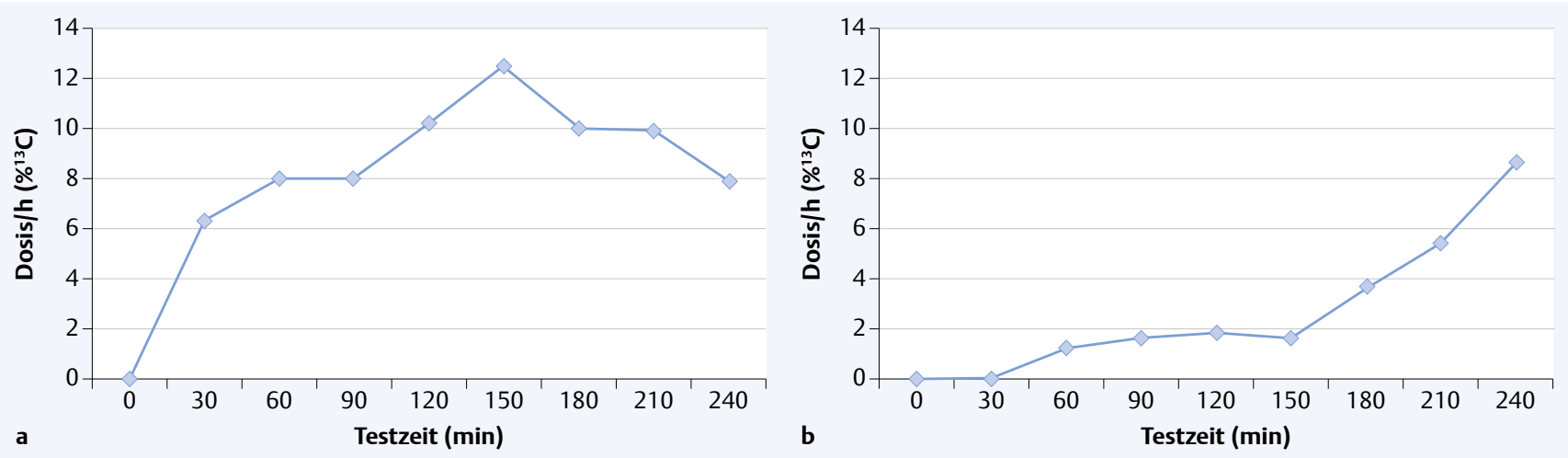

Abb. $3{ }^{13} \mathrm{C}$-Triglycerid-Atemtest a) Zeitgerechte intestinale Fettverdauung mit adäquatem Anstieg der ${ }^{13} \mathrm{C}$-Konzentration in der Ausatemluft. b) Deutlich verzögerte intestinale Fettverdauung mit verzögertem und geringerem Anstieg der ${ }^{13} \mathrm{C}$-Konzentration in der Ausatemluft. Befund passend zu einer exokrinen Pankreasinsuffizienz.

Mahlzeiten eingenommen und die Dosis bedarfsadaptiert gesteigert werden. Solange starke Diarrhöen persistieren, besteht keine Gefahr der Darmatonie. Eine Dosis von $16 \mathrm{mg}$ / Tag sollte nach Möglichkeit jedoch nicht überschritten werden [3].

Racecadotril | Racecadotril bzw. sein aktiver Metabolit Tiorphan ist ein Enkephalinase-Inhibitor und hat dadurch einen antisekretorischen Effekt, im Gegensatz zu Loperamid aber keinen Einfluss auf die Darmtransitzeit [21]. Zugelassen ist Racecadotril in Deutschland für die Behandlung der akuten Diarrhö bei Kindern und Erwachsenen. Zur Langzeitanwendung bei chronischer Diarrhö gibt es allerdings nur wenige Daten.

Colestyramin | Der Gallensäurebinder Colestyramin kann bei einigen Patienten ebenfalls gute Effekte erzielen. Ein Therapieansprechen gilt beim sekundären Gallensäureverlustsyndrom als diagnostischer Nachweis [3].

\section{Therapie des Reizdarmsyndroms mit Diarrhö}

Plausibles Krankheitsmodell vermitteln I In der Therapie des Reizdarmsyndroms ist es notwendig, dem Patienten ein plausibles Krankheitsmodell und ein kongruentes Behandlungskonzept zu vermitteln. Hierbei sollen auch individuelle Triggerfaktoren gezielt eruiert und im Krankheitsmodell und Behandlungskonzept berücksichtigt werden [15]. Zur symptomatischen Therapie stehen Ballaststoffe (Gelbildner) und Probiotika mit positivem Einfluss auf die Stuhlkonsistenz und -frequenz zur Verfügung [22, 23]. Des Weiteren kommen Loperamid und Colestyramin zum Einsatz [24-26].

Low-FODMAP-Diät | Es gibt keine generellen Ernährungsempfehlungen beim Reizdarmsyndrom, allerdings kann eine sogenannte „Low-FODMAPDiät" (fermentable oligo-, di- and monosacchari- des and polyols) eine Symptomlinderung bezüglich Meteorismus, Diarrhö und begleitender Schmerzsymptomatik erzielen. Bei der Low-FODMAP-Diät werden Nahrungsmittel mit einem hohen Anteil an fermentierbaren Oligo-, Di-, Monosacchariden und Polyolen für 6 bis 8 Wochen gemieden.

Die FODMAP sind in nur geringem Maße absorbierte kurzkettige Kohlenhydrate, die osmotisch aktiv sind und nahezu unverändert in den Dickdarm gelangen, dort von Darmbakterien fermentiert werden und zu Diarrhö und Meteorismus führen können.

Nach der initialen Phase von 6 bis 8 Wochen, in der man FODMAP-reiche Nahrungsmittel meidet, folgt eine zweite Phase, in der die verschiedenen FODMAP-haltigen Nahrungsmittel auf ihre individuelle Verträglichkeit getestet werden. Ziel ist es,

- die FODMAP-haltigen Nahrungsmittel, welche im konkreten Einzelfall Beschwerden auslösen, zu erkennen und

- diese langfristig in der Ernährung wegzulassen, ohne dabei zu große diätetische Einschränkungen vorzunehmen.

- Die Beschwerden sollen dabei auf ein erträgliches Maß reduziert werden [27, 28].

Zur Durchführung einer Low-FODMAP-Diät sollte eine Ernährungsberatung erfolgen.

Entspannungsübungen | Hilfreich sein können beim Reizdarmsyndrom

- Entspannungsübungen (nach Jacobson, autogenes Training),

- Selbsthilfestrategien sowie

- bei relevanter psychosozialer Belastung und psychischen Komorbiditäten eine Psychotherapie (kognitive Verhaltenstherapie, psychodynamische Therapie und darmbezogene Hypnose).

Trizyklische Antidepressiva I Niedrigdosiert eingesetzte trizyklische Antidepressiva (z.B. Amitryptilin) können bei Reizdarmsyndrom mit Di- 


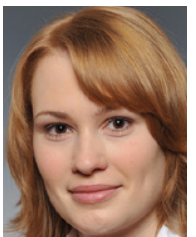

Dr. Korinna Ulbricht ist Assistenzärztin für Innere Medizin am Israelitischen Krankenhaus in Hamburg. k.ulbricht@ik-h.de

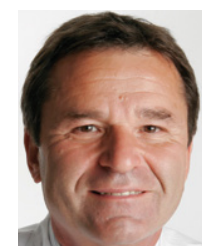

Prof. Dr. Peter Layer ist Ärztlicher Direktor des Israelitischen Krankenhauses sowie Direktor und Chefarzt der Medizinischen Klinik, Israelitisches Krankenhaus, Hamburg. med@ik-h.de

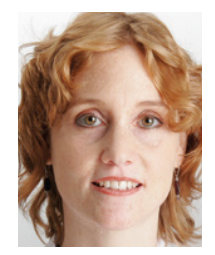

Dr. Viola Andresen, MSc ist Funktionsoberärztin und leitet das Palliativ- und Ernährungsteam am Israelitischen Krankenhaus in Hamburg.

V.Andresen@ik-h.de

Vollständiges Literaturverzeichnis unter

http://dx.doi.

org/10.1055/s-0042-108700

DOI 10.1055/s-0042-108700

VNR2760512016149754008

Dtsch Med Wochenschr

2016; 141: 1395-1402

(c) Georg Thieme Verlag KG .

Stuttgart . New York .

ISSN 0012-0472 arrhö durch Verzögerung der intestinalen Transitzeit positive Effekte auf Stuhlfrequenz und -konsistenz haben. Gleichzeitig wirken sie auf Schmerzen und auf psychische Komorbiditäten (Depression, Angst) [29-31].

Rifaximin I Rifaximin ist in Deutschland zur Therapie der Reisediarrhö und der hepatischen Enzephalopathie zugelassen. Es scheint positive Effekte auf die Stuhlkonsistenz beim Reizdarmsyndrom mit Diarrhö zu haben [32, 33]. Auch allgemeine abdominelle Beschwerden wie Distension, Meteorismus und Flatulenz beim Reizdarmsyndrom mit Diarrhö können sich unter Rifaximin bessern [34-36].

5-HT3-Antagonisten | Die beste Studienlage beim Reizdarmsyndrom mit Diarrhö hinsichtlich der Besserung von Bauchschmerzen und Durchfallsymptomen liegt für 5-HT3-Rezeptor-Antagonisten vor. Als Nebenwirkung tritt vor allem teils starke Obstipation auf [37]. In Deutschland gibt es derzeit keine für die Behandlung des Reizdarmsyndroms zugelassene Substanz. Alosetron ist in den USA nur für Frauen mit therapierefraktären Beschwerden zugelassen, da es in der Vergangenheit zu einzelnen Fällen mit schwerer Obstipation (bis hin zum Ileus) und ischämischer Kolitis gekommen ist. Ramosetron ist nur in Japan, einigen Ländern Südostasiens und Indien erhältlich $[25,38,39]$. Es gibt Anhalt dafür, dass es unter Ramosetron seltener zu ausgeprägter Obstipation als unter Alosetron kommt [38, 40]. Für den in Deutschland verfügbaren 5-HT3-Antagonisten Ondansetron (zugelassen zur Behandlung des Chemotherapie-induzierten Erbrechens) ist ebenfalls eine gute Wirksamkeit bei Reizdarmsyndrom mit Diarrhö belegt [41].

Eluxadolin | In den USA wurde im Mai 2015 von der United States Food and Drug Administration (FDA) der $\mu$ - und к-Opioid-Rezeptor-Agonist und $\delta$-Opioid-Rezeptor-Antagonist Eluxadolin für die Behandlung des Reizdarmsyndroms mit Diarrhö zugelassen. Die Substanz wirkt lokal im enterischen Nervensystem und führt zu einer signifikanten Verbesserung der Stuhlkonsistenz und der abdominellen Schmerzen [36]. Der Zulassungsantrag für Europa ist bei der EMA eingereicht.

Bakterielle Fehlbesiedlung des Dünndarms | Eine bakterielle Fehlbesiedlung des Dünndarms tritt meist postoperativ bei blinden bzw. ausgeschalteten Schlingen, Anastomosenstenosen oder $\mathrm{Zu}$ stand nach Ileozökalresektion auf. Ohne vorausgegangene Operation kann sie auftreten bei

- (Duodenal-)Divertikeln,

- Dünndarmstenosen (M. Crohn, radiogen, Lymphom),

- Immunsuppression,

- Immunglobulinmangel und

- intestinalen Motilitätsstörungen.
Ist eine chirurgische Sanierung möglich, sollte diese erfolgen. Die konservative Therapie kann mit dem darmselektiven Antibiotikum Rifaximin $3 \times 550 \mathrm{mg} /$ Tag für 10 Tage mit relativ wenigen Nebenwirkungen erfolgen. Es handelt sich hierbei allerdings um einen „Off-label-use“. Eine Alternative ist eine rotierende Antibiose (z.B. jeweils 10 Tage Ciprofloxacin $2 \times 250 \mathrm{mg}$, Metronidazol $2-3 \times$ tägl $400 \mathrm{mg}$ und Doxycyclin $1 \times 100 \mathrm{mg}$ ) und die parallele Einnahme von medizinischer Hefe sein [42].

\section{Konsequenz für Klinik und Praxis}

- Warnsymptome wie Fieber, Gewichtsverlust, Blut-, Schleim- oder Eiterbeimengungen im Stuhl, Bauchschmerzen, nächtliche Diarrhö, positive Familienanamnese für CED und kolorektale Karzinome, entfärbter Stuhl sollten aktiv erfragt werden.

- Ferner ist auf Zeichen einer Exsikkose, Malnutrition, Anämie zu achten.

- Warnsymptome sollten im klinischen Kontext immer eine ausführliche Differenzialdiagnostik zur Folge haben.

- Bei der Endoskopie sollten Kolonstufenbiopsien aus allen Kolonabschnitten sowie Biopsien aus Ileum und ggf. Duodenum entnommen werden.

- Zur symptomatischen medikamentösen Behandlung der Diarrhö kommt primär Loperamid, aber auch Colestyramin und Racecadotril zum Einsatz.

- Eine Low-FODMAP-Diät, ballaststoffreiche Kost (Gelbildner) oder Probiotika können beim Reizdarmsyndrom zu einer Symptomlinderung führen.

- In Therapie-refraktären Fällen eines Reizdarmsyndroms mit Diarrhö können Rifaximin, 5-HT3-Antagonisten oder niedrig dosierte trizyklische Antidepressiva helfen.

- Neu und bisher nur in den USA zugelassen für das Reizdarmsyndroms mit Diarrhö sind der $\mu$ - und $\mathrm{k}$-Opioid-Rezeptor-Agonist und $\delta$-Opioid-Rezeptor-Antagonist Eluxadolin.

\section{Interessenkonflikt}

K. U. gibt an, dass kein Interessenkonflikt vorliegt. P.L hat Honorare von AbbVie, Abbott, Almirall, Allergan-Pharm, Falk, Normark, Norgine, Shire, Takeda und Yakult erhalten. V. A. erklärt, dass sie Honorare für Vorträge und/oder Beratungstätigkeiten von Boehringer Ingelheim, Allergan, Almirall, AstraZeneca, Falk, KyowaKirin, Nordmark, Schwabe, Shire und Yakult erhalten hat. 


\section{Literatur}

1 Laux G, Kühlein T, Gutscher A et al. Versorgungsforschung in der Hausarztpraxis - Ergebnisse aus dem CONTENT-Projekt 2006-2009. München: Springer Medizin, Urban und Vogel GmbH, 2010

2 Wilking $\mathrm{H}$, Spitznagel $\mathrm{H}$, Werber $\mathrm{D}$ et al. Acute gastrointestinal illness in adults in Germany: a population-based telephone survey. Epidemiol Infect 2013; 141: 2365-2375

3 Layer P, Rosien U, Thiel S et al. Gallensäureverlustsyndrom. In: Praktische Gastroenterologie. P. Layer, U. Rosien (Hrsg.). 2011, Urban \& Fischer Verlag: München: 157-158

4 Lankisch PG, Mahlke R, Lübbers H et al. Leitsymptom Diarrhö. Dtsch Arztebl 2006; 103: A261-A269

5 Stotzer PO, Abrahamsson H, Bajor A et al. Are the definitions for chronic diarrhoea adequate? Evaluation of two different definitions in patients with chronic diarrhoea. United European Gastroenterol J 2015; 3: 381-386

6 Felber], Aust D, Baas S et al. [Results of a S2kConsensus Conference of the German Society of Gastroenterolgy, Digestive- and Metabolic Diseases (DGVS) in conjunction with the German Coeliac Society (DZG) regarding coeliac disease, wheat allergy and wheat sensitivity]. Z Gastroenterol 2014; 52: 711-743

7 Abraham BP, Kane S. Fecal markers: calprotectin and lactoferrin. Gastroenterol Clin North Am 2012; 41: 483-495

8 Schoepfer AM, Trummler M, Seeholzer P et al. Discriminating IBD from IBS: comparison of the test performance of fecal markers, blood leukocytes, CRP, and IBD antibodies. Inflamm Bowel Dis 2008; 14: 32-39

9 Vermeire S, Van Assche G, Rutgeerts P. Laboratory markers in IBD: useful, magic, or unnecessary toys? Gut 2006; 55: 426-431

10 Roseth AG, Fagerhol MK, Aadland E et al. Assessment of the neutrophil dominating protein calprotectin in feces. A methodologic study. Scand J Gastroenterol 1992; 27: 793-798

11 von Roon AC, Karamountzos L, Purkayastha S et al. Diagnostic precision of fecal calprotectin for inflammatory bowel disease and colorectal malignancy. Am J Gastroenterol 2007; 102 : 803-813

12 Fischer B, Hoh S, Wehler M et al. Faecal elastase-1: lyophilization of stool samples prevents false low results in diarrhoea. Scand J Gastroenterol 2001; 36: 771-774

13 Hoffmeister A, Mayerle J, Beglinger C et al. [S3-Consensus guidelines on definition, etiology, diagnosis and medical, endoscopic and surgical management of chronic pancreatitis German Society of Digestive and Metabolic Diseases (DGVS)]. Z Gastroenterol 2012; 50: 1176-1224

14 Barnert J. Dünndarmfunktionstests. In: Klinische Gastroenterologie - Das Buch für Fort- und Weiterbildung. H. Messmann (Hrsg.). 2011, Georg Thieme Verlag KG: Stuttgart. 61

15 Layer P, Andresen V, Pehl C et al. [Irritable bowel syndrome: German consensus guidelines on definition, pathophysiology and management]. Z Gastroenterol 2011; 49: 237-293

16 Romagnuolo J, Schiller D, Bailey RJ. Using breath tests wisely in a gastroenterology practice: an evidence-based review of indications and pitfalls in interpretation. Am J Gastroenterol 2002; 97 : 1113-1126

17 Posserud I, Stotzer PO, Bjornsson ES et al. Small intestinal bacterial overgrowth in patients with irritable bowel syndrome. Gut 2007; 56: 802-808
18 Corazza GR, Menozzi MG, Strocchi A et al. The diagnosis of small bowel bacterial overgrowth. Reliability of jejunal culture and inadequacy of breath hydrogen testing. Gastroenterology 1990; 98: 302-309

19 Golger D, Scheubel R. Pankreasfunktionstests. In: Klinische Gastroenterologie - Das Buch für Fortund Weiterbildung, H. Messmann, Editor. 2011, Georg Thieme Verlag KG: Stuttgart. 57.

20 Keim V, Klar E, Poll M et al. Postoperative care following pancreatic surgery: surveillance and treatment. Dtsch Arztebl Int 2009; 106: 789-794

21 Eberlin M, Muck T, Michel MC. A comprehensive review of the pharmacodynamics, pharmacokinetics, and clinical effects of the neutral endopeptidase inhibitor racecadotril. Front Pharmacol 2012; 3: 93

22 Brenner DM, Moeller MJ, Chey WD et al. The utility of probiotics in the treatment of irritable bowel syndrome: a systematic review. Am J Gastroenterol 2009; 104: 1033-1049

23 McFarland LV, Dublin S. Meta-analysis of probiotics for the treatment of irritable bowel syndrome. World J Gastroenterol 2008; 14: 2650-2661

24 Wedlake L, A'Hern R, Russell D et al. Systematic review: the prevalence of idiopathic bile acid malabsorption as diagnosed by SeHCAT scanning in patients with diarrhoea-predominant irritable bowel syndrome. Aliment Pharmacol Ther 2009; 30: 707-717

25 Lee KJ. Pharmacologic Agents for Chronic Diarrhea. Intest Res 2015; 13: 306-312

26 Trinkley KE, Nahata MC. Treatment of irritable bowel syndrome. J Clin Pharm Ther 2011; 36: 275-82

27 Shepherd SJ, Halmos E, Glance S. The role of FODMAPs in irritable bowel syndrome. Curr Opin Clin Nutr Metab Care 2014; 17: 605-609

28 Shepherd SJ, Lomer MC, Gibson PR. Short-chain carbohydrates and functional gastrointestinal disorders. Am J Gastroenterol 2013; 108: 707-717

29 Ford AC, Talley N], Schoenfeld PS et al. Efficacy of antidepressants and psychological therapies in irritable bowel syndrome: systematic review and meta-analysis. Gut 2009; 58: 367-378

30 Rahimi R, Nikfar S, Rezaie A et al. Efficacy of tricyclic antidepressants in irritable bowel syndrome: a meta-analysis. World J Gastroenterol 2009; 15 : 1548-1553

31 Gorard DA, Libby GW, Farthing M]. Effect of a tricyclic antidepressant on small intestinal motility in health and diarrhea-predominant irritable bowel syndrome. Dig Dis Sci 1995; 40: 86-95

32 Kane JS, Ford AC. Rifaximin for the treatment of diarrhea-predominant irritable bowel syndrome. Expert Rev Gastroenterol Hepatol 2016; 10: 431-442

33 Thompson JR. Is irritable bowel syndrome an infectious disease? World J Gastroenterol 2016; 22: 1331-1334

34 Sharara Al, Aoun E, Abdul-Baki H et al. A randomized double-blind placebo-controlled trial of rifaximin in patients with abdominal bloating and flatulence. Am J Gastroenterol 2006; 101: 326-333

35 Pimentel M, Park S, Mirocha J et al. The effect of a nonabsorbed oral antibiotic (rifaximin) on the symptoms of the irritable bowel syndrome: a randomized trial. Ann Intern Med 2006; 145: 557-563

36 Rivkin A, Rybalov S. Update on the Management of Diarrhea-Predominant Irritable Bowel Syndrome: Focus on Rifaximin and Eluxadoline. Pharmacotherapy 2016; 36: 300-316 
37 Andresen V, Montori VM, Keller J et al. Effects of 5-hydroxytryptamine (serotonin) type 3 antagonists on symptom relief and constipation in nonconstipated irritable bowel syndrome: a systematic review and meta-analysis of randomized controlled trials. Clin Gastroenterol Hepatol 2008; 6: 545-555

38 Chiba T, Yamamoto K, Sato S et al. Long-term efficacy and safety of ramosetron in the treatment of diarrhea-predominant irritable bowel syndrome. Clin Exp Gastroenterol 2013; 6: 123-128

39 Fukudo S, Kinoshita Y, Okumura T et al. Ramosetron Reduces Symptoms of Irritable Bowel Syndrome With Diarrhea and Improves Quality of Life in Women. Gastroenterology 2016; 150: 358-366 e8
40 Nee J, Zakari M, Lembo AJ. Novel Therapies in IBS-D Treatment. Curr Treat Options Gastroenterol 2015; 13: 432-440

41 Garsed K, Chernova J, Hastings M et al. A randomised trial of ondansetron for the treatment of irritable bowel syndrome with diarrhoea. Gut 2014; 63: 1617-1625

42 Keller J, Layer P, Rosien. Bakterielle Fehlbesiedlung. In Praktische Gastroenterologie. P. Layer, U. Rosien (Hrsg.). 2011; Urban \& Fischer Verlag: München: 164-165 


\section{CME-Fragen}

CME-Teilnahme

- Viel Erfolg bei Ihrer CME-Teilnahme unter http://cme.thieme.de

- Diese Fortbildungseinheit ist 12 Monate online für eine CME-Teilnahme verfügbar.

- Sollten Sie Fragen zur Online-Teilnahme haben, unter http:// cme.thieme.de/hilfe finden Sie eine ausführliche Anleitung.

1. Welche Aussage zur Differenzialdiagnostik bei chronisch nichtinfektiöser Diarrhö trifft nicht zu?

a Die Einnahme von NSAR kann die Aussagefähigkeit des D-XyloseTests zur Bestimmung der Absorptionskapazität des Dünndarms beeinträchtigen

b Bei einer Colitis ulcerosa ist der Schleimhautbefall typischerweise kontinuierlich.

c Reizdarmsymptome können auch Erstsymptome eines Ovarialtumors sein.

d Ein Gallensäureverlustsyndrom lässt sich mittels SeHCAT-Test nachweisen.

e Eine verminderte Pankreas-Elastase $1 \mathrm{im}$ Stuhl spricht gegen eine exokrine Pankreasinsuffizienz.

2. Welche Aussage bezüglich des fäkalen Calprotectins trifft zu?

a Erhöhte Werte sprechen gegen das Vorliegen einer chronisch entzündlichen Darmerkrankung.

b Calprotectin ist nicht hilfreich bei der Unterscheidung einer chronisch entzündlichen Darmerkrankung von einem Diarrhödominanten Reizdarmsyndrom

c Die Calprotectin-Konzentration im Stuhl korreliert mit der Anzahl der in den Gastrointestinaltrakt eingewanderten neutrophilen Granulozyten bei Entzündungsreaktionen.

d Calprotectin ist bei Raumtemperatur sehr instabil und die Bestimmung daher aufwendig.

e Calprotectin ist ein hochspezifischer Marker für eine Colitis ulcerosa.

3. Welches Verfahren dient zur Diagnose einer bakteriellen Fehlbesiedlung des Dünndarms?

a $\mathrm{H}_{2}$-Laktose-Atemtest

b Pankreas-Elastase $1 \mathrm{im}$ Stuhl

c Calprotectin im Stuhl

d $\mathrm{H}_{2}$-Glukose-Atemtest

e SeHCAT-Test

4. Welche Aussage bezüglich eines $\mathrm{H}_{2}$-Fruktose-Atemtests trifft zu?

a Liegt ein $\mathrm{H}_{2}$-Non-Producer-Status vor, kann ein $\mathrm{H}_{2}$-Fruktose-Atemtest trotz gestörter Fruktoseresorption unauffällig ausfallen.

b Eine bakterielle Fehlbesiedlung des Dünndarms hat keinen Einfluss auf das Ergebnis eines $\mathrm{H}_{2}$-Fruktose-Atemtest.

c Ein $\mathrm{H}_{2}$-Laktulose-Atemtest ist zur Evaluation eines möglicherweise vorliegenden $\mathrm{H}_{2}$-Non-Producer-Status als Ursache eines falsch negativen $\mathrm{H}_{2}$-Fruktose-Atemtest-Befundes ungeeignet.

d Ein positiver $\mathrm{H}_{2}$-Fruktose-Atemtest mit Anstieg der $\mathrm{H}_{2}$-Konzentration in der Ausatemluft ist in der Regel nicht geeignet, um eine Fruktosemalabsorption zu diagnostizieren.

e Eine zeitlich unmittelbar vorangegangene orale Einnahme von Antibiotika beeinflusst das Ergebnis eines $\mathrm{H}_{2}$-Atemtest nicht.

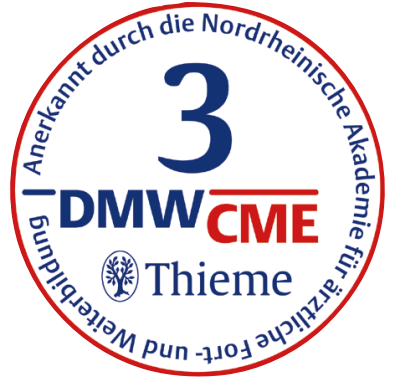

5. Wobei handelt es sich um einen indirekten Funktionstest zur Überprüfung der exokrinen Pankreasfunktion?

a $\mathrm{H}_{2}$-Glukose-Atemtest

b ${ }^{13} \mathrm{C}$-Triglyzerid-Atemtest

c ${ }^{13} \mathrm{C}$-Harnstoff-Atemtest

d $\mathrm{H}_{2}$-Laktulose-Atemtest

e ${ }^{13} \mathrm{C}$-Oktansäure-Atemtest

6. Was eignet sich am besten zur Therapie einer chronischen Diarrhö bei exokriner Pankreasinsuffizienz?

a Loperamid

b Racecadotril

c Pankreasenzympräparate in individueller Dosierung

d Probiotika

e Colestyramin

7. Welche Aussage zur postcibalen Asynchronie trifft zu? Sie kann Ursache einer chronischen Obstipation sein.

Es kommt häufig zu einer Gewichtszunahme.

c Es kommt zu einer unzureichenden Durchmischung von Speisebrei und Pankreasenzymen; ein Teil der Nahrung bleibt unverdaut.

d Sie tritt ausschließlich nach einer Whipple-OP auf.

e Eine Pankreasenzymsubstution hat keinen positiven Effekt auf begleitend auftretende Diarrhö.

8. Welche Aussage zur Low-FODMAP-Diät trifft nicht zu? Eine spezifische Ernährungsberatung zu Beginn ist sinnvoll.

b Nahrungsmittel mit einem hohen Anteil an fermentierbaren Oligo-, Di-, Monosacchariden und Polyolen sollen gemieden werden.

c Reizdarmsyndrombeschwerden können gelindert werden.

d In der initialen Phase werden „FODMAP-reiche“ Nahrungsmittel gemieden. Die zweite Phase dient dazu, die Verträglichkeit verschiedener „FODMAP-haltiger“ Nahrungsmittel auszuprobieren.

e Ziel der Low-FODMAP-Diät ist eine Gewichtsreduktion.

9. Welcher Wirkstoff kann bei einem Reizdarmsyndrom mit Diarrhö zur Beschwerdelinderung eingesetzt werden?

a Natriumpicosulfat

b Linaclotid

c Niedrigdosiert Amitryptilin

d Bisacodyl

e Prucaloprid

10. Welche Bedingungen begünstigen eine bakterielle Fehlbesiedlung des Dünndarmes in der Regel nicht?

a Intestinale Motilitätsstörungen

b Zustand nach Ileozökalresektion

c Duodenaldivertikel

d Sigmaresektion

e Dünndarmstenosen bei Morbus Crohn 


\section{Angaben zur Person}

Name, Vorname, Titel:

Straße, Hausnr.:

PLZ, Ort:

Anschrift: $\square$ privat $\square$ dienstlich

Ich bin Mitglied der Ärztekammer (bitte Namen der Kammer eintragen):

Jahr meiner Approbation:

Ich befinde mich in der Weiterbildung zum:

Ich habe eine abgeschlossene Weiterbildung in ...

(bitte Fach eintragen):

Ich bin tätig als: $\square$ Assistenzarzt $\square$ Oberarzt $\square$ Chefarzt $\square$ niedergelassener Arzt $\square$ Sonstiges Ich bin DMW-Abonnent: $\square$ ja $\square$ nein

Falls nein: ich habe den Fragebogen aus / von:

$\square$ Thieme-connect $\square$ Kollegen $\square$ der Klinik $\square$ einer Bibliothek $\square$ Sonstiges

Lernerfolgskontrolle (Eine Antwort pro Frage ankreuzen)
1. $\square$ A $\square$ B $\square$ C $\square$ D $\square$ E
2. $\square$ A $\square$ B $\square \mathrm{C} \quad \square$ D $\square$ E
3. $\square$ A $\square$ B $\square$ C $\square$ D $\square$ E
4. $\square$ A $\square$ B $\square$ C $\square$ D $\square$ E
5. $\square$ A $\square$ B $\square$ C $\square$ D $\square$ E
6. $\square$ A $\square$ B $\square$ C $\square$ D $\square \mathrm{E}$
7. $\square$ A $\square$ B $\square$ C $\square$ D $\square$ E
8. $\square$ A $\square$ B $\square$ C $\square$ D $\square$ E
9. $\square \mathrm{A} \quad \square \mathrm{B} \quad \square \mathrm{C} \quad \square \mathrm{D} \quad \square \mathrm{E}$
10. $\square \mathrm{A} \quad \square$ B $\square \mathrm{C} \quad \square \mathrm{D} \quad \square \mathrm{E}$

Ich versichere, dass ich die Beantwortung der Fragen selbst und ohne fremde Hilfe durchgeführt habe

Ort, Datum:

Unterschrift:

Bitte in dieses Feld Ihre DMW Abonnement-Nummer eintragen:

\section{Fragen zur Zertifizierung}

1. Das Thema des Beitrages kommt in meiner ärztlichen Tätigkeit $\square$ häufig vor $\square$ selten vor $\square$ regelmäßig vor $\square$ gar nicht vor

2. Bei diesem Thema habe ich $\square$ eine feste Gesamtstrategie $\square$ keine Strategie $\square$ noch offene Einzelprobleme

3. In Bezug auf das Thema des Beitrages

$\square$ fühle ich mich nach dem Studium des Beitrags in meiner Strategie bestätigt

$\square$ habe ich meine Strategie verändert:

$\square$ habe ich erstmals eine einheitliche Strategie erarbeitet

$\square$ habe ich keine einheitliche Strategie ableiten können

4. Wurden aus der Sicht Ihrer täglichen Praxis heraus wichtige Aspekte des Themas

nicht erwähnt: $\square$ ja, welche $\square$ nein

zu knapp abgehandelt? $\square$ ja, welche $\square$ nein

überbewertet? $\square$ ja, welche $\square$ nein

5. Verständlichkeit des Beitrages

$\square$ Der Beitrag ist nur für Spezialisten verständlich

$\square$ Der Beitrag ist auch für Nicht-Spezialisten verständlich

6. Beantwortung der Fragen

$\square$ Die Fragen lassen sich aus dem Studium des Beitrags allein beantworten

$\square$ Die Fragen lassen sich nur unter Zuhilfenahme zusätzlicher Literatur beantworten

7. Die Aussagen des Beitrages benötigen eine ausführlichere Darstellung

$\square$ zusätzlicher Daten

$\square$ von Befunden bildgebender Verfahren

$\square$ die Darstellung ist ausreichend

8. Wieviel Zeit haben Sie für das Lesen des Beitrages und der Bearbeitung des Quiz benötigt?

Zertifizierungsfeld (wird durch die DMW ausgefüllt)

Ihr Ergebnis:

Sie haben von 10 Fragen richtig beantwortet.

Sie haben $\square$ bestanden und 3 Punkte erworben $\square$ nicht bestanden $\square$ ungültig, weil:

Stuttgart, den Stempel/Unterschrift 